\author{
Vittorio Di Piero \\ Novella Bonaffini \\ Marta Altieri
}

\section{Migraine and cerebrovascular disease}

\begin{abstract}
Migraine and cerebrovascular disease are linked in different ways: migraine may be a potential cause of stroke as in migrainous infarction, headache may be a symptom of cerebrovascular disease and also a risk factor for stroke, the association of migraine and stroke may constitute specific syndromes such as CADASIL and MELAS. The new IHS 2003 criteria, though preserving their main structure, have changed the terminology regarding secondary headaches, now described as "attributed to" another disease rather than "associated with" it. The more detailed knowledge of causal links between the underlying disorder and headache, has allowed to strengthen the terminology. Many cerebrovascular disorders as cerebral haemorrhage, venous sinus thrombosis, carotid or vertebral dissections and ischaemic stroke may present with a headache or be followed by it. In subarachnoid haemorrhage (SAH) headache may constitute an important warning sign before the bleeding. An interesting issue is the hypothesis that migraine may be a potential risk factor for stroke. Recent studies have underlined the increased
\end{abstract}

relative risk of ischemic stroke in female migraineurs. Many potential mechanisms have been hypothesized: (1) alterations of vasoreactivity due to vessel wall dysfunction, (2) release of vasoactive substances during migraine, (3) platelet hyperactivity as expression of serotoninergic dysfunction in migraineurs, (4) intriguing studies have described a high prevalence of migraine with aura in stroke patients with patent foramen ovale (PFO). Differential diagnosis between migraine and stroke remains fundamental: some types of migraine can mimic cerebrovascular disease such as familial hemiplegic migraine, and basilar migraine. Migraine and stroke may be part of syndromic complexes as in CADASIL and MELAS. In conclusion migraine is a risk factor for cerebrovascular disease, it may be the cause of stroke as in migrainous infarctions, stroke may induce headache which may be a relevant symptom of cerebrovascular disease, yet migraine remains an essentially benign condition.

Key words Migraine $\cdot$ Cerebrovascular disease $\cdot$ IHS criteria
Migraine and cerebrovascular disease are linked in different ways: (1) migraine may be the cause of cerebrovascular disease as in migrainous infarction; (2) headache may be a symptom of cerebrovascular disease; (3) migraine may be a risk factor for ischaemic stroke, especially when associated with specific habits; (4) 
migraine may be part of, in association with stroke, specific syndromes such as CADASIL (cerebral autosomal dominant arteriopathy with subcortical infarcts and leucoencephalopathy) and MELAS (mitochondrial encephalopathy, lactic acidosis and stroke-like episodes).

In the new International Headache Society (IHS) 2003 criteria [1], the definition of migrainous infarction (described as one or more migrainous aura symptoms, lasting longer than $60 \mathrm{~min}$, associated with an ischaemic brain lesion in an appropriate territory demonstrated by neuroimaging) is unchanged from the previous version. In order to diagnose a migrainous infarction the symptoms must occur during a migraine attack.

The new criteria, although basically preserving the main structure of the previous version, have changed the terminology regarding secondary headaches, which are now described as "attributed to" another disease rather than by the former, less precise, term "associated with". More detailed knowledge, in most cases, of the causal links between the underlying disorder and the headache has led to more accurate terminology. In particular, in secondary headaches, the criteria underline how headache during cerebrovascular events may present in various forms and in some cases resemble migraine even though the characteristics of the headache itself are still poorly described in the scientific literature [1].

A number of cerebrovascular disorders may present with a headache or be followed by it, including cerebral haemorrhage, venous sinus thrombosis, carotid or vertebral dissections and ischaemic stroke. In subarachnoid haemorrhage (SAH), headache may precede other symptoms by days and constitute an important warning sign before the haemorrhage.

Headache presenting during stroke is often secondary to serious conditions with a potentially bad outcome such as cerebral haemorrhage or $\mathrm{SAH}$; headache is often a sign of worsening of cerebral oedema in ischaemic and haemorrhagic stroke, quite different from migrainous infarction which has a better prognosis.

An interesting aspect of migraine and cerebrovascular disease is the hypothesis that the latter may be a potential risk factor for stroke. It is nowadays widely accepted that, especially in young women, migraine with aura may be considered a risk factor for stroke, in particular when associated with a smoking habit and the use of oral contraceptives. Recent studies have underlined the increased relative risk of ischaemic stroke in female migraineurs - especially if smokers or using oral contraceptives - reaching an odds ratio ranging, according to different studies, from 1.2 to 16.3 [2-5].
A number of potential mechanisms have been hypothesized to lead from migraine to stroke: (1) alterations of vasoreactivity and of cerebral blood flow due to vessel wall dysfunction; (2) release of vasoactive substances during migraine (such as prostaglandins, endothelin, etc.); (3) platelet hyperactivity as a potential expression of serotoninergic dysfunction in migraineurs, although study results are controversial on this issue [6]; and (4) a high prevalence of migraine with aura in stroke patients with patent foramen ovale (PFO), recently described in intriguing studies [7-9]. In support of the involvement of PFO in migraine with aura, its surgical closure has been associated with improvement of migraine [10].

All the above-mentioned issues have a clinical relevance in the choice of therapeutic strategies and in the prevention of cerebrovascular disease, which must be considered especially in young women who are smokers and take oral contraceptives. The role of migraine as a risk factor for cerebrovascular disease looses significance with increasing age [11].

Differential diagnosis between migraine and stroke remains an important issue: there are types of migraine that can mimic cerebrovascular disease and which must, therefore, be considered when approaching a patient presenting with an acute focal deficit. Familial hemiplegic migraine is a hereditary autosomal dominant disease, due to a mutation on chromosome 19 , in which headache is preceded and sometimes accompanied by hemiparesis. The motor weakness lasts from 30 to $60 \mathrm{~min}$, but other neurological defects such as blurred speech, nystagmus and ataxia may persist between attacks. Basilar migraine mimics a vertebrobasilar stroke presenting with an aura which may consist in visual defects, dysarthria, vertigo, tinnitus, ataxia, decreased level of consciousness or diplopia.

Two main diseases have been described in which migraine and stroke are part of the syndromic complex which defines the conditions. In CADASIL, white matter abnormalities and subcortical infarcts are associated with cognitive decline and a history of migraine. In MELAS, a mitochondrial myopathy is associated with leucoencephalopathy, stroke-like episodes and headache with the characteristics of migraine [12].

In conclusion, migraine is a risk factor for cerebrovascular disease; it may be the cause of stroke as in migrainous infarctions, and stroke may induce headache which may be a relevant symptom of cerebrovascular disease. However, migraine remains an essentially benign condition. 


\section{References}

1. Headache Classification Subcommittee of the International Headache Society (2004) The international classification of headache disorders, 2nd edn. Cephalalgia 24 [Suppl 1]

2. Chang CL, Donaghy M, Pouolter N, and the World Health Organisation Collaborative Study of Cardiovascular Disease and Steroid Hormone Contraception (1999) Migraine and stroke in young women: case-control study. BMJ 318:13-18

3. Tzourio C, Iglesias S, Hubert JB, et al (1993) Migraine and risk of ischaemic stroke: a case-control study. BMJ 307:289-292

4. Salobir B, Sabovic M, Peternel P, Stegnar M, Grad A (2002) Classic risk factors, hypercoagulability and migraine in young women with cerebral lacunar infarctions. Acta Neurol Scand 105:189-195
5. Bousser MG, Conrad J, Kittner S, de Lignieres B, MacGregor EA, Massiou H, Silberstein SD, Tzourio C (2000) Recommendations on the risk of ischaemic stroke associated with use of combined oral contraceptives and hormone replacement therapy in women with migraine. The International Headache Society Task Force on Combined Oral Contraceptives \& Hormone Replacement Therapy. Cephalalgia 20:155-156

6. Crassard I, Conard J, Bousser MG (2001) Migraine and haemostasis. Cephalalgia 21:630-636

7. Anzola GP, Magoni M, Guindani M, Rozzini L, Dalla Volta G (1999) Potential source of cerebral embolism in migraine with aura. A transcranial Doppler study. Neurology 52:1622-1630

8. Sztajzel R, Genoud D, Roth S, Mermillod B, LeFloch-Rohr J (2003) Patent foramen ovale, a possible cause of symptomatic migraine: a study of 74 patients with acute ischemic stroke. Headache 43:305-306
9. Lamy C, Giannesini C, Zuber M, Arquizan C, Meder JF, Trystram D, Coste J, Mas JL for the Patent Foramen Ovale and Atrial Septal Aneurysm Study Group (2002) Clinical and Imaging findings in cryptogenic stroke patients with and without patent foramen ovale. The PFO-ASA study. Stroke 33:706-711

10. Wilmshurst PT, Nightningal S, Walsh KP, Morrison WL (2000) Effect on migraine of closure of cardiac right-toleft shunts to prevent recurrence of decompression illness or stroke or for haemodynamic reasons. Lancet 356:1648-1651

11. Mosek A, Marom R, Korczyn A, Bornstein N (2001) A history of migraine is not a risk factor to develop an ischemic stroke in the elderly. Headache 41:399-401

12. Dichgans M (2002) CADASIL: A monogenic condition causing stroke and subcortical vascular dementia. Cerebrovasc Dis 13[Suppl 2]:37-41 